\title{
Eine Trendanalyse zur zeitlichen Entwicklung von Qualitätsunterschieden zweier Versorgungsformen auf Basis von Sekundärdaten
}

\author{
Secondary Data-based Trend Analysis of How Quality Differences \\ in Two Forms of Healthcare Develop Over Time
}

\section{Autoren}

Olga A. Sawicki ${ }^{1}$, Anastasiya Glushan ${ }^{1}$, Angelina Müller ${ }^{1}$, Martin Beyer ${ }^{1}$, Kateryna Karimova ${ }^{1},{ }^{*}$ Renate Klaaßen-Mielke²,

Institute

1 Institut für Allgemeinmedizin, Goethe-Universität Frankfurt am Main, Frankfurt am Main, Deutschland

2 Institute of Medical Informatics, Biometry and Epidemiology, Ruhr-Universität Bochum, Bochum, Deutschland

\section{Schlüsselwörter}

Sekundärdatenanalyse, Trendanalyse, Versorgungsforschungsanalyse, Versorgungsprogramme, medizinische, kompetitive

\section{Key words} secondary data analysis, trend analysis, health services research analysis, Competitive Health Plan

online publiziert $\quad 25.10 .2021$

\section{Bibliografie}

Gesundheitswesen 2021; 83 (Suppl. 2): S97-S101

DOI 10.1055/a-1630-7086

ISSN 0949-7013

(C) 2021. Thieme. All rights reserved.

Georg Thieme Verlag, Rüdigerstraße 14,

70469 Stuttgart, Germany

\section{Correspondence}

Dr. Olga A. Sawicki

Institut für Allgemeinmedizin,

Goethe-Universität Frankfurt am Main

Theodor-Stern-Kai 7

60326 Frankfurt am Main

Deutschland

sawicki@allgemeinmedizin.uni-frankfurt.de

$\Theta$

Zusätzliches Material finden Sie unter https://doi.org/ 10.1055//a-1630-7086

geteilte Letztautorenschaft

\section{ZUSAMMENFASSUNG}

Ziel der Studie Die Evaluation komplexer Interventionen wie Versorgungsformen ist methodisch herausfordernd. Ziel dieser Analyse ist anhand generalisierter Schätzungsgleichungen (GEE) zu prüfen, wie sich die Qualitätsunterschiede zwischen der Hausarztzentrierten Versorgung (HZV) und der Regelversorgung über die Zeit entwickeln.

Methoden Auf der Basis von Routinedaten der AOK BadenWürttemberg wurde eine längsschnittliche Sekundärdatenanalyse der Jahre 2011 bis 2018 durchgeführt. Versicherte mit passenden Einschlusskriterien wurden im Sinne einer dynamischen Kohorte zu Beginn des jeweiligen Beobachtungsjahres in die Analyse eingeschlossen, sodass der Umfang und die Zusammensetzung der Kohorte von Jahr zu Jahr variierten. Mithilfe von GEE wurden Qualitätsunterschiede in dichotomen Indikatoren zwischen der HZV und der Regelversorgung untersucht, wobei für Mehrfachmessungen eine autoregressive Kovarianzstruktur (AR1) zugrunde gelegt wurde. An Variablen wurden berücksichtigt: Eine Gruppenvariable für die Versorgungsformen, eine Variable für das betrachtete Jahr sowie eine Wechselwirkung zwischen den beiden Variablen. Die aus der Modellierung resultierenden Schätzer lieferten Auskunft darüber, wie das Verhältnis der beiden Gruppen zu Beginn der Beobachtungsstudie war und wie sich die Gruppentrends sowohl individuell als auch im Verhältnis zueinander über die Zeit entwickelten.

Ergebnisse GEE wird beispielhaft auf den Qualitätsindikator Verordnung von potenziell inadäquater Medikation (PIM) bei älteren Versicherten angewandt. Die Chance einer PIM-Verordnung bei über 65-jährigen (Jahr 2018; N = 628 523) war zu Beginn der Beobachtungszeit innerhalb der HZV signifikant niedriger als in der Regelversorgungsgruppe (Odds Ratio 0,978; $95 \%$-Konfidenzintervall: 0,968-0,987). In beiden Gruppen nahm die Chance einer PIM-Verordnung in den betrachteten 7 Beobachtungsjahren ab, in der HZV-Gruppe stärker als in der Regelversorgungsgruppe.

Schlussfolgerung Eine Trendanalyse mit GEE basierend auf Sekundärdaten, die die Qualitätsunterschiede zwischen Vergleichsgruppen abbildet, bietet großes Potenzial zur Evaluation neuer und bestehender Versorgungsformen über die Zeit. 


\section{ABSTRACT}

Aim of the study The evaluation of complex interventions such as different forms of healthcare are methodologically challenging. The aim of this study was to use generalized estimating equations (GEE) to investigate how quality differences between family practitioner-based healthcare (HZV) and standard care develop over time.

Methods A longitudinal secondary data analysis for 20112018 was conducted using routine data from the AOK Baden Wuerttemberg health insurer. A dynamic cohort of insured persons that fulfilled the inclusion criteria were included in the analysis at the beginning of each year of observation, so the size and composition of the cohorts varied from year to year. Quality differences between HZV and standard care were investigated, whereby an autoregressive covariance structure (AR1) was assumed for multiple measurements. Under observation were a group variable for healthcare type, a variable for year of observation, and any interaction between the two. The resulting estimates provided information on the relationship between the two groups at the beginning of the observation period, and on how the groups developed both individually and in relation to one another over time.

Results The GEE were used exemplarily on the quality indicator prescription of potentially inappropriate medication (PIM) in elderly insured patients. At the beginning of the observation period, the chance of PIM in those over 65 years of age (year 2018; $N=628,523$ ) was significantly lower in the HZV group than in the group receiving standard care (odds ratio 0.978; $95 \%$ confidence interval: $0.968-0.987$ ). The chance of a PIM in the following seven years declined in both groups, but faster in the HZV group than the group receiving standard care.

Conclusion A secondary data-based trend analysis with GEE of quality differences in comparison groups over time has considerable potential in the evaluation of new and existing forms of healthcare.

\section{Einleitung}

Das deutsche Gesundheitssystem wird im internationalen Vergleich als durchschnittlich beurteilt [1]. Um die Leistungsfähigkeit zu erhöhen, wurde mit der Gesundheitsreform im Jahr 2000 die Möglichkeit der Gestaltung besonderer Versorgungsformen ( $§ 140$ a des fünften Sozialgesetzbuches) erweitert. Besondere Versorgungsformen können sich jedoch nur durchsetzen, wenn ihr Mehrwert mit belastbaren Evaluationsergebnissen nachgewiesen werden kann [2]. Schwerpunkte der Versorgungsforschung liegen dabei in der Beschreibung der Versorgungssituation und der Trends unter Alltagsbedingungen, um Fehlentwicklungen zu erklären und Optimierungspotentiale aufzuzeigen [3]. In Deutschland werden Versorgungsformen jedoch selten evaluiert. Bei der Evaluierung von Versorgungsformen ist die Frage, inwieweit die Versorgungsqualität tatsächlich auch langfristig verbessert wird, entscheidend. Einen Goldstandard zur Einschätzung der Versorgungsqualität im zeitlichen Verlauf gibt es derzeit nicht. Häufig werden Patienten- bzw. Ärztebefragungen durchgeführt. Diese Methoden sind anfällig für systematische Verzerrungen und gehen mit einem hohen Erhebungsaufwand einher [4]. Die Nutzung von Sekundärdaten ist indes besonders vorteilhaft, da die Forschungsfrage keinen Einfluss auf die Daten hat. Administrative Datenbanken der gesetzlichen Krankenversicherungen (GKV) sind dabei aufgrund des Stichprobenund Variablenumfangs sowie der zeitnahen Verfügbarkeit eine wichtige Datenquelle. Durch die Datenverfügbarkeit über mehrere Jahre und die Verknüpfung versichertenbezogener Daten mit Informationen aus unterschiedlichen Leistungssektoren haben sich neue Möglichkeiten für die Längsschnittdatenanalyse ergeben [5].

Das longitudinale Studiendesign ist in der Versorgungsforschung weit verbreitet, da es sich besonders für die Analyse von Qualitätsentwicklungen eignet. Dieses Design beinhaltet wiederholte Messungen, bei denen für ein Individuum mehrere Beobachtungen zu unterschiedlichen Zeitpunkten erfasst werden und somit eine Abhängigkeit innerhalb der Messwiederholungen entsteht. Diese Korrelation sollte adäquat berücksichtigt werden. Die von
Liang und Zeger [6] eingeführten generalisierte Schätzungsgleichungen (englisch generalized estimating equations, GEE) bieten dabei die Möglichkeit, die Abhängigkeitsstruktur der Messungen zu berücksichtigen.

Ziel dieser Studie ist anhand der GEE-Methode die Qualitätsunterschiede zwischen den Versorgungsformen der Hausarztzentrierten Versorgung (HZV) und der Regelversorgung hinsichtlich dichotomer Zielgrößen über die Zeit abzubilden. Diese werden hier aus Platzgründen beispielhaft anhand des Qualitätsindikators der Verordnung von potenziell inadäquater Medikation (PIM) bei älteren Versicherten dargestellt.

\section{Methoden}

\section{Datengrundlage}

Auf der Basis von Routinedaten der AOK Baden-Württemberg als größten regionalen Anbieter der HZV wurde eine Längsschnittanalyse durchgeführt. Der Datenpool umfasst pseudonymisierte Daten über Ärzte, Betriebsstätten, Versicherte, Pflegegrad sowie die Teilnahme an der HZV und an den Disease Management Programmen. Weiterhin enthält der Pool Daten zu Leistungsausgaben der stationären und ambulanten Behandlungen, Arzneimittelabrechnungsdaten, Verordnungen sowie Diagnosen. Für die Analyse standen Daten der Jahre 2011 bis 2018 zur Verfügung. Die Rohdaten wurden an das aQua-Institut übermittelt und die Datenkonsistenz überprüft. Betrachtet wurden Versicherte, die die Ein-und Ausschlusskriterien zu Beginn des jeweiligen Beobachtungsjahres erfüllten (Supplement Abb. 1). Der Umfang und die Zusammensetzung der Kohorte variierten daher von Jahr zu Jahr (dynamische Kohorte).

Außerdem identifizierte das aQua-Institut den Index-Hausarzt nach einem gesondert festgelegten Algorithmus. Auf Basis einer vordefinierten Indikatorenliste bereitete das aQua-Institut Ausgangsdatensätze mit Zielvariablen vor. 


\section{Vergleichsgruppen}

Die Versorgungform der HZV und der Regelversorgung wurden im Vergleich betrachtet. Die HZV wurde zur Stärkung der hausärztlichen Primärversorgung durch den Gesetzgeber eingeführt ( $\S 73 \mathrm{~b}$ des fünften Sozialgesetzbuches). Die AOK Baden-Württemberg hat die HZV bereits im Jahr 2008 angefangen zu implementieren. Für die Auswertung erfolgte die Gruppenzuordnung zur HZV-Gruppe, wenn Versicherte in der $\mathrm{HZV}$ eingeschrieben waren und eine hausärztliche Leistung in Anspruch genommen hatten; Versicherte wurden in die Gruppe der Regelversorgung zugeordnet, wenn sie einen nicht an der HZV teilnehmenden Hausarzt besuchten und eine hausärztliche Leistung in der Regelversorgung in Anspruch genommen wurde.

\section{Potenziell inadäquate Medikation (PIM)}

Die Arzneimittelversorgung von älteren Menschen ist häufig durch eine Polypharmazie geprägt, die das Risiko für PIM erhöhen kann [7]. Datengrundlage für die Auswertung des Qualitätsindikators Verordnung von PIM bei älteren Versicherten waren alle zu Lasten der AOK Baden-Württemberg ausgestellten Rezepte, die über öffentliche Apotheken abgerechnet wurden. Als ältere Versicherte wurden Personen jenseits des 65. Lebensjahres betrachtet. Die Medikamente wurden mittels der deutschen PIM-Liste, der PRISCUS-Liste, gewählt [8]. Die PRISCUS-Liste führt 83 Wirkstoffe aus 18 Arzneistoffklassen auf, die für ältere Menschen potenziell inadäquat sind. Zur Operationalisierung wurden diesen Wirkstoffen die jeweiligen ATC-Codes zugeordnet.

\section{Statistische Analyse}

Neben der deskriptiven Auswertung wurden in einer Trendanalyse die Qualitätsunterschiede der beiden Versorgungsformen hinsichtlich dichotomer Zielgrößen, hier speziell der Verordnung von PIM bei älteren Versicherten, herausgearbeitet. Die Parameterschätzungen erfolgen mithilfe von GEEs unter Annahme einer Binomialverteilung und mit einer Logit-Link-Funktion. Dazu war neben der Modellspezifikation die Festlegung der Abhängigkeitsstruktur (unabhängige, austauschbare, AR1 oder unstrukturierte) erforderlich, für die hier eine autoregressive Kovarianzstruktur angenommen wurde (AR1). Diese eignet sich besonders gut, falls es sich um äquidistante Messzeitpunkte handelt. An Einflussvariablen wurden eine dichotome Gruppenvariable (X1 mit $1=\mathrm{HZV}$ und $0=$ Regelversorgung), die Zeitperiode als stetige Variable $(X 2=0,12,3, .$. als Jahr nach Beginn der Beobachtungsperiode) und eine Interaktion aus diesen beiden Variablen (X1 ${ }^{*}$ X2) berücksichtigt. Die resultierenden Schätzer lieferten die Auskunft, wie das Chancenverhältnis (Odds Ratio) der beiden Gruppen (HZV versus Regelversorgung zu Beginn der Beobachtungsperiode war $(\exp (\beta 1)$ und wie sich das Chancenverhältnis sowohl in der Regelversorgung (exp( $\beta 2$ pro Beobachungsjahr) als auch in der HZV im Verhältnis zur Regelversorgung $(\exp (\beta 3)$ über die Zeit entwickelten. Um die Vergleichbarkeit zwischen den Gruppen sicherzustellen, erfolgte zusätzlich eine Adjustierung für Versicherten-und Praxismerkmale, die jährlich erfasst wurden (Supplement Tabelle 2, Im Internet). Das Modell stellte sich wie folgt dar:

$$
Y=\operatorname{logit}(p)=\log (p /(1-p))=\beta_{0}+\beta_{1}{ }^{*} X 1+\beta_{2}{ }^{*} X 2+\beta_{3}{ }^{*} X 1{ }^{*} X 2^{\text {a }}
$$

a Gekürzte Darstellung der Regressionsgleichung ohne Adjustierungsvariablen sowie den zugehörigen Regressionsparametern
Das p gab dabei die Wahrscheinlichkeit für das Eintreten des Ereignisses an.

Aus den Modellberechnungen wurde für eine standardisierte Kohorte eine Grafik zur Visualisierung des zeitlichen Trends erzeugt, indem für die marginale Verteilung der Kovariaten in der zugrundeliegenden Stichprobe die sich aus dem Modell ergebenden Kleinstquadrateschätzer(LSM) für die Anteile der Patienten mit PIMVerordnung pro Berichtsjahr in den Gruppen jeweils durch eine Trendlinie verbunden wurden.

Die Spezifikation des Modells beruht grundsätzlich auf der Annahme, dass es einen Trend gibt. Um zu prüfen, ob diese Annahme gerechtfertigt ist, wurde in einer Sensitivitätsanalyse ein weiteres Modell angepasst, in der die Zeitvariable unter Verwendung von Dummyvariablen für das jeweilige Berichtsjahr als kategoriale Variable berücksichtigt wurde und dadurch beliebige Entwicklungen über die Zeit in den beiden Vergleichsgruppen modelliert werden konnten. Mithilfe der LSM aus der Sensitivitätsanalyse, die ebenfalls in die Grafik aufgenommen wurden, kann visuell beurteilt werden, ob das Modell mit der Annahme eines Trends für die Analyse geeignet ist.

Die Studie folgte der Standardisierten Berichtsroutine für Sekundärdaten

Analysen (STROSA). Für die Auswertungen wurde SAS in der Version 9.4 (SAS Institute Inc., Cary, NC, USA) verwendet. Das Datenmanagement wurde mit IBM-SPSS in der Version 25 (SPSS Inc., Chicago, IL, USA) umgesetzt.

\section{Ergebnisse}

Beispielhaft für das Beobachtungsjahr 2018 sind die Charakteristika der insgesamt 628.523 aus den Routinedaten identifizierten Versicherte $\geq 65$ Jahre in der Supplement Tab. 1, Im Internet dargestellt.

\section{Deskriptiv}

Der Anteil älterer Versicherter mit einer PIM-Verordnung war im Jahr 2011 in der Regelversorgungsgruppe höher als in der HZV ( $\triangleright$ Tab. 1). Im Beobachtungsjahr 2018 blieb diese Tendenz bestehen, bei einem beobachteten Rückgang des Anteils von Patienten mit PIM-Verordnungen insgesamt.

\section{Trendanalyse}

Die Supplement Tabelle 3, Im Internet stellt die Ergebnisse der GEE-Analyse dar. Die multivariable Modellierung zeigt über den gesamten Beobachtungszeitraum einen Vorteil der HZV-Gruppe gegenüber der Regelversorgung. Zu Beginn des Beobachtungszeitraums 2011 war die Chance einer PIM-Verordnung in der HZVGruppe signifikant niedriger als in der Regelversorgungsgruppe (Odds Ratio 0,978 pro Jahr; $95 \%$-Konfidenzintervall 0,968-0,987). Im Verlauf zeigt sich für die Regelversorgung eine abnehmende Chance für eine PIM-Verordnung (Odds Ratio 0,928 pro Jahr; 95\%-Konfidenzintervall 0,927-0,930). In der HZV-Gruppe war die Abnahme stärker ausgeprägt als in der Regelversorgungsgruppe (Odds Ratio 0,986 pro Jahr; $95 \%$-Konfidenzintervall 0,984-0,988). In der $>$ Abb. 1 wird das Ergebnis der Modellberechnungen visualisiert. Der Unterschied zwischen den Gruppen findet sich im Abstand zwischen den Linien wieder. Die erkennbare Veränderung 
> Tab. 1 Deskriptive Statistik der älteren Versicherten $\geq 65$ Jahre der AOK Baden-Württemberg mit mindestens einer Verordnung von potenziell inadäquater Medikation.

\begin{tabular}{|c|c|c|c|}
\hline Ältere Versicherte & HZV & Regelversorgung & Gesamt \\
\hline $\mathrm{n}(2011)$ & 293135 & 282228 & 575363 \\
\hline $\mathrm{n}(2018)$ & 399804 & 228719 & 628523 \\
\hline Verordnung von potenziell inadäquater Medikation in 2011 & $86548(29,5 \%)$ & $86284(30,6 \%)$ & $172832(30,0 \%)$ \\
\hline Verordnung von potenziell inadäquater Medikation in 2018 & $83775(21,0 \%)$ & $50387(22,0 \%)$ & $134162(21,3 \%)$ \\
\hline
\end{tabular}

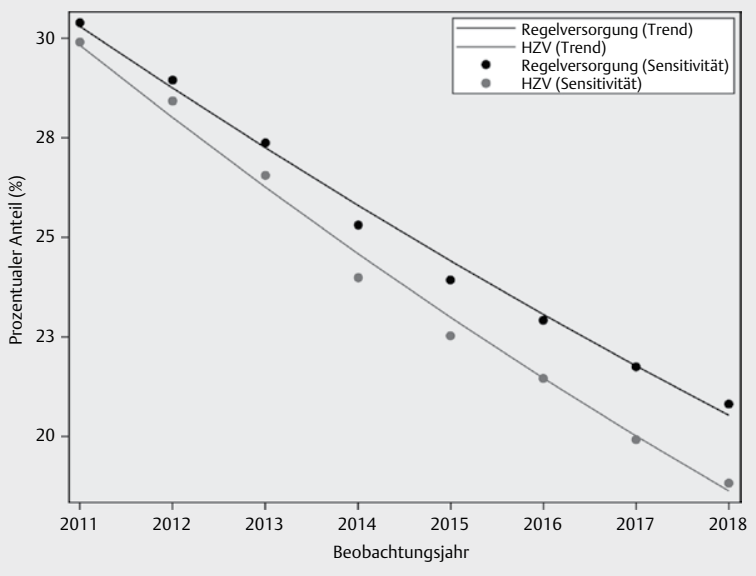

Abb. 1 Ergebnisse der Längsschnittanalyse: Vorhersagen für Anteile der älteren Versicherten $\geq 65$ Jahre der AOK Baden-Württemberg mit mindestens einer Verordnung einer potenziell inadäquaten Medikation in einer standardisierten Kohorte für die Hausarztzentrierte Versorgung (HZV) sowie Regelversorgung.

dieses Abstands weist auf die unterschiedliche Entwicklung in den Gruppen über die Beobachtungszeit hin. Die Geradendarstellung ergibt sich aus den getroffenen Modellannahmen. Die Sensitivitätsanalyse zeige, dass die Trendannahme gerechtfertigt erscheint.

\section{Diskussion}

Ziel der vorliegenden Studie war die Darstellung der zeitlichen Entwicklung von Qualitätsunterschieden zwischen der HZV und Regelversorgung auf Basis von Sekundärdaten. Das angewandte GEEModell enthält 3 Parameter: Die Gruppenvariable HZV und die Trendvariablen Gesamttrend in der Regelversorgungsgruppe sowie Relativer Trend der HZV-versus Regelversorgungsgruppe. Dies erlaubt sowohl die Analyse des Verhältnisses der beiden Versorgungsformen zu Beginn der Studie als auch die Entwicklung der Gruppentrends individuell und im Verhältnis zueinander über die Zeit zu beschreiben. Die Untersuchung der GKV-Versichertendaten bzgl. des hier exemplarisch dargestellten Qualitätsindikators ergab sowohl zu Beobachtungsbeginn als auch im zeitlichen Trend eine statistisch signifikante niedrigere PIM-Verordnungschance bei den über 65-jährigen in der HZV als in der Regelversorgung. Darüber hinaus zeigen die Auswertungen einen allgemeinen Rückgang der PIM-Verordnung. Die Ergebnisse der Längsschnittanalysen weiterer Qualitätsindikatoren sind unter [9] zu finden. Es konnte konstatiert werden, dass die Versorgungsform der HZV in Baden-Würt- temberg mit ihrer strukturierten und koordinierten Versorgung nachhaltig zur Versorgungsqualität beiträgt.

Die hiesige Betrachtung einer offenen Kohorte über mehrere Zeitperioden führt zur Verletzung der für die einfache logistische Regression notwendigen Annahme der Unabhängigkeit zwischen den Beobachtungen. Es gibt unterschiedliche Methoden zur Berücksichtigung der Abhängigkeitsstruktur. [6, 10,11]. Der einfachste Ansatz ist die Ancova mit Messwiederholung, die allerdings vollständige Messungen für jeden Messzeitpunkt voraussetzt, was im Widerspruch zur offenen Kohorte steht. Die Berücksichtigung unvollständiger Messreihen ist auch in generalisierten linearen gemischten Modellen (GLMM) möglich. Im Gegensatz hierzu weisen GEEs auch bei falsch spezifizierter Korrelationsstruktur den Vorteil auf, zuverlässige Parameterschätzungen und Standardfehler zu liefern [12]. Da sich zudem aus diesem Modellansatz populationsgemittelte Schätzer ergeben [13], bieten GEEs großes Potenzial zur Bewertung komplexer Interventionen wie neue Versorgungsformen. Da das GEE-Modell auf der Theorie der großen Stichprobe basiert [13], empfehlen wir ihre Anwendung bei ausreichend großen Stichproben. Abschließend ist anzumerken, dass das GEE-Model unter Anpassung der Link-Funktion auch für stetige oder Zählindikatoren geeignet ist.

Als Limitationen sind zu nennen, dass die Analyse ausschließlich abgerechnete Arzneimittel berücksichtigt. Eine zusätzliche Selbstmedikation ist nicht auszuschließen. Des Weiteren waren klinische Daten nicht vorhanden und ein Rückgriff auf Rezeptdaten oder Patientenakten nicht möglich. Auch Self-Selection Bias war sowohl bei den Hausärzten als auch bei den Versicherten in der HZV-Gruppe nicht auszuschließen. Ferner wäre eine zusätzliche externe Validierung der Krankenkassendaten z. B. Vollständigkeit und Korrektheit von Kodierungen und Verschlüsselungen wie ATC erstrebenswert. Insgesamt bieten unsere Auswertungen einen guten Einblick in die PIM-Verordnung im GKV-System. Es lassen sich jedoch keine Aussagen für den privaten Gesundheitssektor treffen.

Der GEE-Ansatz auf Basis der hier verwendeten sektorenübergreifenden Routinedaten der AOK Baden-Württemberg lässt eine solide Darstellung der Qualitätsunterschiede zweier Versorgungsformen unter Alltagsbedingungen über einen längeren Zeitraum zu.

\section{Danksagung}

Wir danken der AOK Baden-Württemberg für die Bereitstellung der Daten. Ebenfalls danken wir Dr. Claudia Witte aus dem aQUA Institut für das Datenmanagement und Phillip Elliott für die Übersetzung der Zusammenfassung ins Englische. Rolle der Sponsoren: Die Evaluation der HZV Baden-Württemberg wurde durch die AOK Ba- 
den-Württemberg, den Hausärzteverband Baden-Württemberg und MEDI Baden-Württemberg gefördert.

\section{Interessenkonflikt}

O.A.S., A.G., A.M., M.B. und K.K. haben über die Goethe-Universität Mittel der AOK-Baden-Württemberg erhalten. R.K.-M. gibt keine Interessenskonflikte an.

\section{Literatur}

[1] World Health Organization. The world health report 2000: Health systems; improving performance. Geneva: World Health Organization

[2] Wiethege J, Ommen O, Ernstmann N et al. Transparenz als Voraussetzung für Innovation in der Versorgungsforschung: Defizite am Beispiel der Evaluation von Managed-Care-Modellvorhaben. Gesundheitswesen 2010; 72: 722-728. doi:10.1055/s-0029-1239567

[3] Glaeske G, Augustin M, Abholz H et al. Epidemiologische Methoden für die Versorgungsforschung. Gesundheitswesen 2009; 71: 685-693

[4] Swart E, Stallmann C, Powietzka J et al. Datenlinkage von Primär-und Sekundärdaten: Ein Zugewinn auch für die kleinräumige Versorgungsforschung in Deutschland? Bundesgesundheitsblatt Gesundheitsforschung Gesundheitsschutz 2014; 57: 180-187. doi:10.1007| s00103-013-1892-1

[5] Swart E, Ihle P. Epidemiologische Studien auf Basis von Sekundärdaten. Public Health Forum 2012; 20: 16-17. doi:10.1016/j.phf.2012.06.016
[6] Liang K-Y, Zeger SL. Longitudinal Data Analysis Using Generalized Linear Models. Biometrika 1986; 73: 13. doi:10.2307/2336267

[7] Bao Y, Shao H, Bishop TF et al. Inappropriate medication in a national sample of US elderly patients receiving home health care. J Gen Intern Med 2012; 27: 304-310. doi:10.1007/s11606-011-1905-4

[8] Holt S, SchmiedI S, Thürmann PA. Potentially inappropriate medications in the elderly: the PRISCUS list. Dtsch Arztebl Int 2010; 107: 543-551. doi:10.3238/arztebl.2010.0543

[9] Universitätsklinikum Heidelberg, Institut für Allgemeinmedizin Goethe Universität Frankfurt. Evaluation des Hausarztzentrierten Versorgung (HZV) in Baden-Württemberg. 2020; Im Internet: https://www.aok.de/ gp/fileadmin/user_upload/Arzt_Praxis/Aerzte_Psychotherapeuten/ Vertraege_Vereinbarungen/Hausarztzentrierte_Versorgung/ Baden-Wuerttemberg/HZV-Evaluation_Broschuere_2020.pdf; Stand: 26.03.2021

[10] McCullagh P, Nelder JA. Generalized linear models. $2^{\text {nd }}$ ed. London: Chapman \& Hall; 1999

[11] Kleinbaum DG, Klein M. Logistic Regression for Correlated Data: GEE. In: Kleinbaum DG, Klein M, Rihl Pryor E. eds. Logistic regression: A self-learning text. $3^{\text {rd }}$ ed. New York, NY: Springer; 2010: 489-538

[12] Fitzmaurice GM, Laird NM, Ware JH. Applied Longitudinal Analysis. $2^{\text {nd }}$ ed. Hoboken: Wiley; 2012

[13] Wang M, Li C-S. Generalized Estimating Equations in Longitudinal Data Analysis: A Review and Recent Developments. Advances in Statistics 2014; 2014: 303728. doi:10.1155/2014/303728* in Memoriam Prof. Dr. Dieter Felsenberg 